\title{
The Constitutive Endopolygalacturonase TvPG2 Regulates the Induction of Plant Systemic Resistance by Trichoderma virens
}

\author{
Sabrina Sarrocco, Fabiola Matarese, Riccardo Baroncelli, Giovanni Vannacci, Verena Seidl-Seiboth, \\ Christian Peter Kubicek, and Mariarosaria Vergara
}

First, second, third, fourth, and seventh authors: Department of Agriculture, Food and Environment, University of Pisa, Italy, via del Borghetto 80, 56124 Pisa, Italy; fifth and sixth authors: Research Area Biotechnology and Microbiology, Institute of Chemical Engineering, TU Wien, 1060 Vienna, Austria; and seventh author: Scuola Normale Superiore di Pisa, piazza dei Cavalieri 7, 56126 Pisa, Italy.

Accepted for publication 10 January 2017.

ABSTRACT

\begin{abstract}
Trichoderma spp. are opportunistic fungi some of which are commonly present in the rhizosphere. Several species, such as T. virens, are also efficient biocontrol agents against phytopathogenic fungi and exert beneficial effects on plants. These effects are the consequence of interactions between Trichoderma and plant roots, which trigger enhanced plant growth and induce plant resistance. We have previously shown that $T$. virens I10 expresses two endopolygalacturonase genes, tvpg1 and tvpg2, during the interaction with plant roots; tvpg 1 is inducible while tvpg2 is constitutively transcribed. Using the same system, the tomato polygalacturonase-inhibitor gene Lepgipl was induced at the same time as tvpg1. Here we show by gene disruption that TvPG2 performs a regulatory role on the inducible
\end{abstract}

tvpg1 gene and in triggering the plant immune response. A tvpg2-knockout strain fails to transcribe the inducible tvpgl gene in neither in vitro in inducing media containing pectin or plant cell walls, nor during the in vivo interaction with tomato roots. Likewise, the in vivo induction of Lepgip1 does not occur, and its defense against the pathogen Botrytis cinerea is significantly reduced. Our data prove the importance of a $T$. virens constitutively produced endopolygalacturonase in eliciting plant induced systemic resistance against pathogenic fungi.

Additional keywords: gene knockout, polygalacturonase inhibiting proteins.
Trichoderma is a fungal genus originating from mycoparasites that evolved into opportunistic fungi. It is able to colonize diverse habitats that harbor living organisms including plants (Druzhinina et al. 2011; Kubicek et al. 2011). Due to their mycoparasitic abilities, many species have been reported as suitable for the biological control of fungal plant pathogens (Benitez et al. 2004; Hermosa et al. 2012; Kubicek et al. 2001). In addition, the ability of some isolates to colonize the plant rhizosphere, and thereby to trigger plant resistance and enhanced plant growth, has fostered practical applications (De Meyer et al. 1998; Salas-Marina et al. 2011; Velázquez-Robledo et al. 2011). In the interaction with plants they can colonize the first or second layers of the root cell walls but, unlike plant pathogens, usually do not penetrate further (Harman et al. 2004). They thus induce metabolic changes in the plants (Fiorini et al. 2016), which can cause systemic resistance to a wide range of pathogens (Shoresh et al. 2010), and sometimes also lead to growth promotion (SalasMarina et al. 2011).

Examples are $T$. asperellum and $T$. harzianum which induce systemic resistance and plant defense responses in cucumber (Elsharkawy et al. 2013; Yedidia et al. 1999, respectively). T. virens can enhance biomass and root growth in Arabidopsis (ContrerasCornejo et al. 2009), and induces phytoalexin production and resistance to Rhizoctonia solani in cotton roots (Howell et al. 2000), and to Botrytis cinerea in Arabidopsis (Velázquez-Robledo et al. 2011). Such plant responses are triggered by fungal proteins that come into contact with the plant cell wall (Kubicek et al. 2011, 2014; Mukherjee et al. 2012).

Plant cell wall degrading enzymes, such as xylanases, were the first fungal proteins that were found to induce such a reaction (Lotan and Fluhr 1990). In plant-pathogenic fungi, pectin degrading

Corresponding author: S. Sarrocco; E-mail address: sabrina.sarrocco@unipi.it

(c) 2017 The American Phytopathological Society enzymes, notably endopolygalacturonases (EPGs), act as virulence factors because they are produced in the early stages of contact with plant tissues (Annis and Goodwin 1997; Di Pietro and Roncero 1998). EPGs, together with pectin lyases, can disrupt the middle lamella of the plant and consequently macerate the plant tissue (Herron et al. 2000), which is a prerequisite for penetration into the host and for using it as a carbon source. The importance of this step for plant-pathogenic fungi is also reflected in the presence in plants of polygalacturonase inhibiting proteins (PGIPs) (Federici et al. 2001), and by the fact that oligogalacturonides - formed by the polygalacturonases-elicit the plant defense reactions (Cervone et al. 1989).

Although polygalacturonases are much more abundant in the genomes of plant pathogenic fungi, saprotrophic fungi also contain several polygalacturonase genes to feed on decaying plant material (Kubicek et al. 2014; Zhao et al. 2013). The genomes of Trichoderma spp. contain up to four polygalacturonase genes (Kubicek et al. 2011), but their potential role as plant elicitors has not been extensively investigated. One canonical EPG gene from the T. harzianum strain T34, which is induced upon contact with the plant, and is present in all Trichoderma spp. (C. P. Kubicek, unpublished data), has been reported to be involved in beneficial associations with plants (MoránDiez et al. 2009).

T. virens strain I10 is an efficient biocontrol agent (Catalano et al. 2011; Sarrocco et al. 2006, 2009; Vannacci and Pecchia 2000). We have previously shown that the transcripts of two EPG genes (tvpg 1 and tvpg2) were highly abundant in this strain during the in vivo association with tomato roots. Data also suggested an EPG-PGIP recognition in that system (Baroncelli et al. 2016). Interestingly, the expression of tvpg 1 was inducible, whereas tvpg 2 was constitutive. Constitutively expressed genes usually represent the core of an organism's physiological inventory because they encode essential proteins involved in basal pathways or processes. Constitutive EPGs may release pectin degradation products, which can induce other EPG encoding genes (Wubben et al. 1999). 
In this study, we further investigated the role of tvpg2, and then of tvpgl during the interaction of $T$. virens with tomato. This entailed exploiting reverse genetics to investigate whether TvPG2 would elicit a defense response against plant pathogens in their host, such as an induced systemic resistance (ISR), using B. cinerea and tomato plants as a model.

\section{MATERIALS AND METHODS}

Fungal strains, culture conditions, and plant material. The $T$. virens I10 isolate (CBS 116947) was used as the wild-type strain. The strain B14 (T. virens I10 $\Delta t k u 70$, Catalano et al. 2011) was used to delete the tvpg 2 gene, since the disruption of $t k u 70$ strongly increases the homologous recombination efficiency. B. cinerea (SAS 56) was kindly provided by F. Faretra (Department of Soil Science, Plant and Food, Bari University, Italy). All fungi were maintained on potato dextrose agar (PDA; Difco, NJ), under mineral oil, at $4^{\circ} \mathrm{C}$, in the fungal collection of the Plant Pathology \& Mycology Laboratory of the Department of Agriculture, Food and Environment (Pisa University). The fungi were grown on PDA plates at $24^{\circ} \mathrm{C}$, with a daily cycle of $12 \mathrm{~h}$ light and $12 \mathrm{~h}$ dark. When needed, fungal liquid cultures in a basal mineral Fries medium containing glucose or apple pectin (Sigma Aldrich, Milan, Italy) or tomato cell walls, all at $0.5 \%$ (wt/vol), were set up as described in Baroncelli et al. (2016).

Tomato seeds, Solanum lycopersicum 'Micro-Tom', were provided by Nicky's Nursery Ltd., Kent, U.K. (www.nickys-nursery.co.uk).

Deletion of tvpg 2 and transformation. In order to disrupt tvpg2 (ID 192705 in the T. virens Gv29-8 genome sequence database v2.0, http://genome.jgi.doe.gov/TriviGv29_8_2/TriviGv29_8_2.home. html), $1.32 \mathrm{~kb}$ of its $5^{\prime}$ noncoding and $1.45 \mathrm{~kb}$ of its $3^{\prime}$ noncoding regions were amplified by PCR. The $5^{\prime}$ upstream region was amplified with the primer pair $\mathrm{A} / \mathrm{B}$ (the forward A containing the restriction site SpeI, the reverse B with a BamHI site); the $3^{\prime}$ downstream region was amplified with the primer pair C/D (the forward C with a HindIII site, the reverse D with a XhoI site) (Table 1). Both regions were cloned into a pGEM-T easy vector (Promega, Madison, WI). The hygromycin phosphotransferase B gene, $h p h B$, conferring resistance to hygromycin $\mathrm{B}$, was excised as a BamHI/HindIII fragment from pLHhph (Hartl et al. 2007), whereas $P d m \mathrm{I} / X m n \mathrm{I}$ were used to linearize the vector. First the PCR upstream (SpeI/BamHI), then the downstream (HindIII/XhoI), fragment and finally the $h p h B$ resistance marker $(2.4 \mathrm{~kb}$ between up- and downstream regions BamHI/HindIII) were ligated into the vector pBluescript SK, resulting in the tvpg2 knockout vector pSGEPG $(8.1 \mathrm{~kb})$. The right orientation of fragments inside the deletion cassette $(5.2 \mathrm{~kb})$ was checked by PCR with the primer pair A/D (Table 1), using the Expand Long Template PCR System (Roche, Indianapolis). The 5.2-kb cassette was used to delete the tvpg2 gene in T. virens I10 4 tku70 (B14) strain. The transformation was carried out with B14 protoplasts, as previously described by Catalano et al. (2011).

PCR and Southern blot analysis of transformants. Fungal genomic DNA was isolated from transformants and control strains I10 and B14 as described in Hartl and Seiboth (2005). Southern blot analysis was carried out following standard protocols (Sambrook and Russell 2001). Probe labeling and hybridization were performed with the DIG nonradioactive system according to the manufacturer's instructions (Roche). A specific probe, including both flanking regions of tvpg 2 and the $h p h B$ gene, was amplified from the pSGEPG DNA with the primer pair A/D (Table 1) and used on genomic DNA digested by EcoRV.

A PCR analysis was performed with the Expand Long Template PCR System (Roche) according to the manufacturer's instructions. Genomic DNA was amplified with the primer pair A/D (Table 1). All primers were provided by MWG-BIOTECH (Ebersberg, Germany).

Physiological analysis of transformants. Fungal growth rates were quantified in 96-well microplates (PbI International, Milan, Italy) in potato dextrose broth (Difco, NJ), using a spectrophotometric approach (Catalano et al. 2011). OD $_{595}$ values were plotted against time to create growth curves, which were then subjected to analysis of variance (ANOVA) for regression, in order to compare the slope of each curve, assuming $P \leq 0.05$ as a significant level. The experiment was independently replicated three times.

To quantify conidial germination, three spore suspensions $\left(10^{3}\right.$ conidia $\cdot \mathrm{ml}^{-1}$ ) were prepared for each strain grown on PDA and used to evaluate the germination ability, as described by Catalano et al. (2011). Data were submitted to one-way ANOVA, assuming $P \leq$ 0.05 as a significant level. Three independent biological replicates were made, each consisting of three slides for each strain. Forty conidia were counted for each slide.

For in vitro confrontation tests the antagonistic and mycoparasitic abilities of transformants were tested in dual cultures against $B$. cinerea as described by Catalano et al. (2011). In order to compare the growth of the pathogen in the presence/absence of the antagonist (wild type or transformants), growth curves on PDA were subjected to ANOVA for regression using Systat (SPSS Inc., Chicago). $P \leq 0.05$ was assumed as a significant level. Three independent replicates were made for each antagonist/pathogen combination.

On the same plates, after 21 days, overgrowth and sporulation of the antagonists on $B$. cinerea colonies were assessed. Interaction zones and overlapping regions for each antagonist/pathogen combination were then analyzed on water agar (agar $15 \mathrm{~g} \cdot$ liter $^{-1}$ of distilled water) by microscopic investigations in order to detect possibly occurring coilings of $T$. virens strains around the host hyphae.

TABLE 1. Primers used for transformation and reverse transcription-PCR

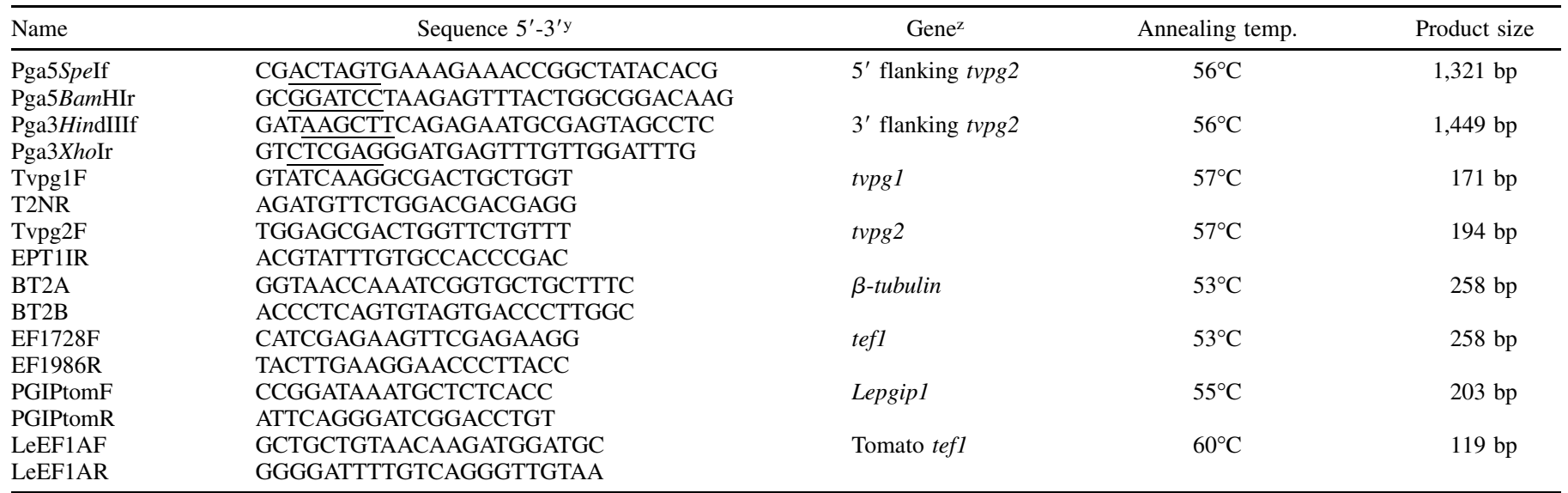

y Underlined nucleotides correspond to target sites of restriction enzymes.

${ }^{\mathrm{z}}$ tef1: translation elongation factor 1-alpha. 
RNA isolation and expression analysis by one step reverse transcription (RT)-PCR. Samples obtained from fungal liquid cultures containing glucose or pectin or tomato cell walls were collected after 48, 72, and $96 \mathrm{~h}$. Total RNAs were then isolated following a guanidinium isothiocyanate/phenol-chloroform method (Baroncelli et al. 2016).

A one step RT-PCR analysis was performed using $1 \mu \mathrm{g}$ of RNA from each sample, according to the manufacturer's instructions (AccessQuick RT-PCR System, Promega, Madison, WI). The primers used are listed in Table 1.

Quantitative RT-PCR. Tomato roots were inoculated by T. virens strains in order to monitor the expression of fungal EPG genes tvpg1 and tvpg2 (accession numbers KF963272 and KF963273, respectively) and the tomato EPG inhibitor gene Lepgipl (accession number L26529). Experimental conditions for in vivo root colonization, total RNA isolation, primers (Table 1) and protocols for quantitative RT-PCR (qRT-PCR) were those described by Baroncelli et al. (2016). Two biological replicates were carried out for each experimental condition. Specific primers used in this experiment were as follows: for T. virens tvpg $1 \mathrm{E} / \mathrm{F}$, for tvpg $2 \mathrm{G} / \mathrm{H}$, and for S. lycopersicum Lepgip1 M/N. The following primers targeting housekeeping genes were used as a reference: for $T$. virens both $\beta$-tubulin $\mathrm{I} / \mathrm{J}$, and the translation elongation factor 1 -alpha tefl $\mathrm{K} / \mathrm{L}$; for tomato the translation elongation factor $L e E F 1 A$ O/P. All primers, supplied by MWG-BIOTECH, are listed in Table 1. Three internal technical replicates were made for each of the two biological replicates.

In vivo biocontrol assay. Tomato seeds were sterilized for 5 min with $50 \% \mathrm{NaClO}$ ( $1 \%$ active chlorine) in a solution ( $\mathrm{vol} / \mathrm{vol})$ of $50 \%$ ethanol and then rinsed for 5 min each three times with distilled water. A pregermination 3-day step was performed in a humid chamber in the dark at $4^{\circ} \mathrm{C}$. The germinated seeds were then transferred at room temperature to pots containing a commercial plant growing substrate (Universal soil Esselunga, supplied by Berco Srl, Calcinate, Bergamo, Italy). Four days later, the soil around the plantlets was preinoculated by $0.5 \mathrm{ml}$ of $T$. virens spore suspensions $\left(10^{6}\right.$ conidia $\left.\cdot \mathrm{ml}^{-1}\right)$ or by water alone (control). After additional 7 days, a $B$. cinerea suspension containing $10^{6}$ conidia $\cdot \mathrm{ml}^{-1}$ was sprayed on all leaves $(0.5 \mathrm{ml} /$ plant $)$ of preinoculated and untreated plants (B. cinerea control). Both control and inoculated plants were evaluated for the presence of foliar symptoms 2 weeks after inoculation. The disease was quantified by counting the number of necrotic lesions developed on each inoculated true leaf. The experiment consisted of two pots with three plants each per treatment, and was performed three times. ANOVA was carried out using Systat (SPSS Inc.).

Root and rhizosphere colonization test. The same experimental scheme used for the biocontrol assay was employed for the root and rhizosphere colonization test. Seven days after the $T$. virens preinoculation (in the biocontrol assay, B. cinerea was also inoculated at the same time), treated and control (untreated) tomato roots were collected. The roots were gently shaken to detach loosely adherent plant growing substrate and were then rinsed with $20 \mathrm{ml}$ of sterile distilled water, and the washing water was collected. This represented the rhizosphere suspension. The roots were then washed again under tap water and sterilized as described in the previous section for seeds, except for the sterilization time which was reduced to $1 \mathrm{~min}$. Each root was drained on sterile filter paper and divided into four to five pieces which were laid on Trichoderma semiselective medium (TSM: PDA amended with 50 ppm of streptomycin sulfate, 7,500 i.u. (international unit) $\cdot$ liter $^{-1}$ bacitracin sulfate, and $0.3 \mathrm{~g} \cdot$ liter $^{-1}$ hymexazol). At the same time, $200 \mu \mathrm{l}$ of the rhizosphere suspension from each root was spread on the surface of a TSM plate. All plates were incubated for up to 2 weeks at room temperature in order to detect the presence of Trichoderma colonies emerging from root tissues or from the rhizosphere. Whenever T. virens developed out of at least one piece of each root, that root was considered as colonized. As an additional control, particles of plant growing substrate used for potting plants were scattered over the surface of TSM plates (about $0.1 \mathrm{~g}$ per plate, 18 plates) and incubated for up to 2 weeks at room temperature.
To verify the presence/absence of $T$. virens, Trichoderma colonies arising from roots, rhizosphere and plant growing substrate (as a control) were isolated on TSM plates. They were then compared with $T$. virens I10 reference colonies of the same age grown on TSM.

\section{RESULTS}

Generation and verification of $T$. virens tvpg 2 knockout mutants. The gene to be deleted was the $T$. virens constitutive EPG tvpg2 (Baroncelli et al. 2016). A 5.2-kb deletion cassette, including a 5' tvpg 2 upstream region (1.32 kb), the $h p h B$ marker $(2.4 \mathrm{~kb})$ and a $3^{\prime}$ tvpg2 downstream region $(1.45 \mathrm{~kb})$, was inserted into the final pSGEPG vector and used for transformation. In order to facilitate the integration of the vector $\mathrm{pSGEPG}$ into the native tvpg 2 locus, we made use of a $T$. virens mutant (B14) derived from the I10 isolate which bears a knocked out $t k u 70$ gene responsible for ectopic integration (Catalano et al. 2011). The transformation and the selection for mitotically stable, hygromycin resistant transformants resulted in three strains $(2,8$, and 12$)$ with the tvpg 2 gene potentially replaced.

To prove the knockout in these transformants, they were analyzed by PCR with primers including the tvpg $25^{\prime}$ and $3^{\prime}$ flanking regions (A/D in Table 1) comparing them to wild-type I10 and I10 $\Delta t k u 70$ B14 strains. The expected molecular amplicon weights, $4.0 \mathrm{~kb}$ in the wild-type and B14, and $5.2 \mathrm{~kb}$ (displaced tvpg 2 + cassette) in mutants were observed (Fig. 1A), confirming the deletion event in all transformants. To verify the integration of the vector into only a single site of the mutants' genomes, an EcoRV Southern blot analysis was performed, since this enzyme cuts at 5' and $3^{\prime}$ tvpg 2 flanking regions, which were both inserted in the deletion cassette. The probe was obtained by PCR on the plasmid pSGEPG with A/D primers (including the deletion cassette). The results were consistent with expectations: a 2.8-kb fragment (unaltered tvpg2, 2,829 bp) in I10 wild type and in B14, and a 4.1-kb fragment (deleted tvpg2, $4,061 \mathrm{bp}$ ) in knockout strains were found, indicating a single site insertion in the transformants' genomes (Fig. 1B).

Phenotype comparison of the knockout strains. Since the presence of the knocked out $t k u 70$ locus in the transformants impaired all attempts to complement the deleted tvpg 2 strains, we used three independent transformants $(2,8$, and 12) in order to assess their basal physiological properties.

Fungal growth rates. After regression analysis of growth curves (all highly significant, $R^{2} \geq 0.95$ and $P<0.0001$ ), no differences

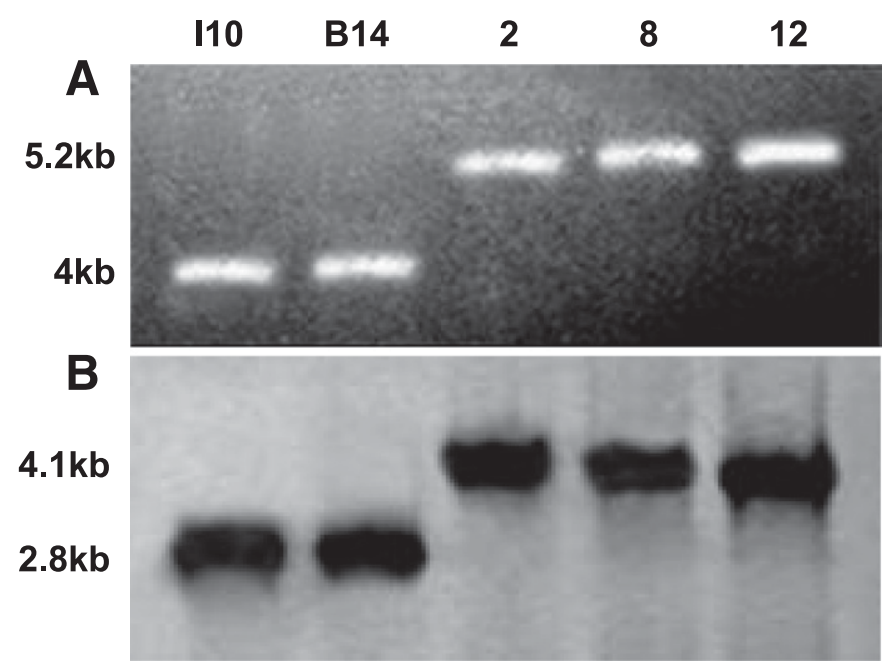

Fig. 1. Molecular analyses of transformants. I10: Trichoderma virens wildtype strain; B14: I10 $\Delta K u 70$ strain; 2, 8, and 12: tvpg2 knockout transformants derived from B14. A, PCR with $\mathrm{A} / \mathrm{D}$ primers external to the tvpg2 coding region. B, Southern blot with genomic DNAs restricted by EcoRV and a probe containing the deletion cassette. 
between the parent strains I10 or B14 and the three mutant strains were detected ( $\left.P_{\text {slope }} \geq 0.108\right)$ (Fig. 2A).

Conidial germination. Spore germination percentages were submitted to ANOVA, after angular transformation, to compare I10 and B14 with the three transformants. Efficiency of germination was not statistically altered in 2 and 12 compared with strain I10, but 8 showed a significant reduction in the percentage of germination. No differences were recorded between B14 and the three transformants (Fig. 2B).

In vitro confrontation tests. The ability of the three mutants to antagonize and mycoparasitize $B$. cinere $a$ was evaluated and compared with $\mathrm{I} 10$ and B14, previously reported as antagonist against this pathogen (Catalano et al. 2011). When growth curves of the pathogen in dual cultures were analyzed, all mutant strains significantly inhibited the growth of $B$. cinerea $\left(P_{\text {slope }} \leq 0.002\right)$ (Fig. 2C). After 21 days, all transformants sporulated on the pathogen, thus indicating mycoparasitism. No differences were apparent among the three knockout strains and I10 or B14 (Fig. 2D). No coilings around B. cinerea hyphae were observed, neither by the wild-type I10 and B14, in agreement with Catalano et al. (2011), nor by any of the transformants.

Because of the consistency in the analyzed phenotypes, a single mutant (number 2) was selected for further analyses, hereafter $\Delta t v p g 2$.

In vitro expression analysis in response to pectin or plant cell walls. In order to check the expression of tvpgl and tvpg 2 genes on different carbon sources, the strains I10, B14 and $\Delta t v p g 2$ were grown in liquid cultures amended with glucose as a control and two potentially inducing substrates, pectin and tomato cell walls. Samples were collected at 48,72, and $96 \mathrm{~h}$ after inoculation and analyzed by reverse transcription PCR. No differences were seen in the expression shown by the parent strains I10 and B14 (Fig. 3A and $\mathrm{B}$, respectively), which is in accordance with the pattern reported
G48 G72 G96 P48 P72 P96 W48 W72 W96

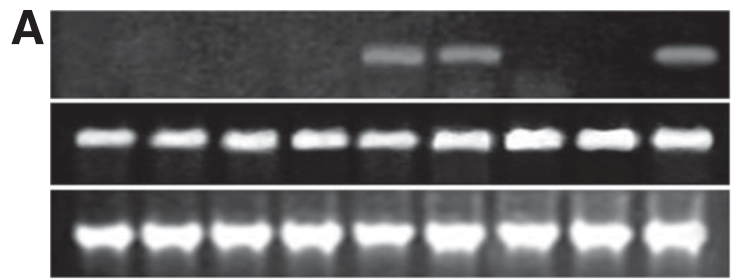

B
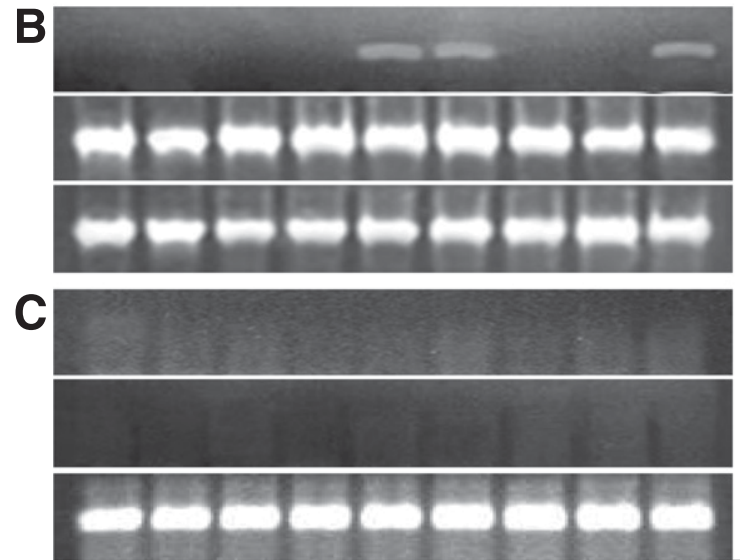

Fig. 3. Reverse transcription-PCR patterns of I10, B14, and $\Delta t v p g 2$ in response to pectin or plant cell walls. G48, G72, and G96: glucose cultures after 48, 72, and $96 \mathrm{~h}$; P48, P72, and P96: pectin cultures at the same times; W48, W72, and W96 plant cell wall cultures at the same times. A, I10; B, B14; C, $\Delta$ tvpg2. For each isolate, the first line corresponds to E/F primers for tvpgl (171 bp), the second line to $\mathrm{G} / \mathrm{H}$ primers for tvpg2 (194 bp), and the third line to $\mathrm{K} / \mathrm{L}$ primers for tefl $(258 \mathrm{bp})$ as a reference gene.
A

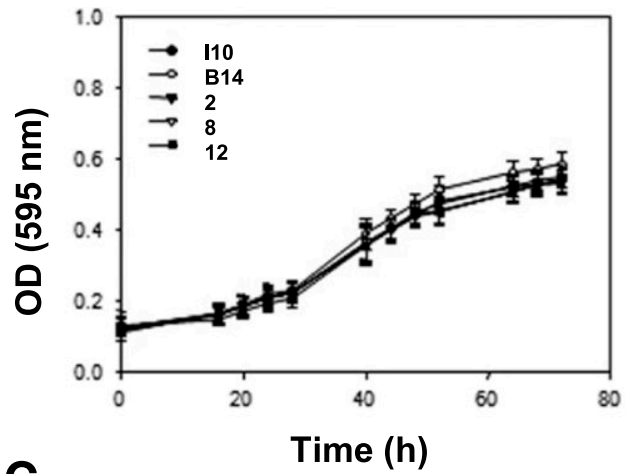

C

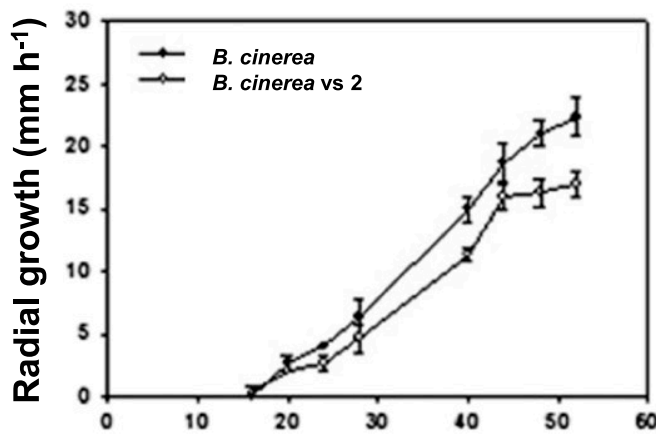

B

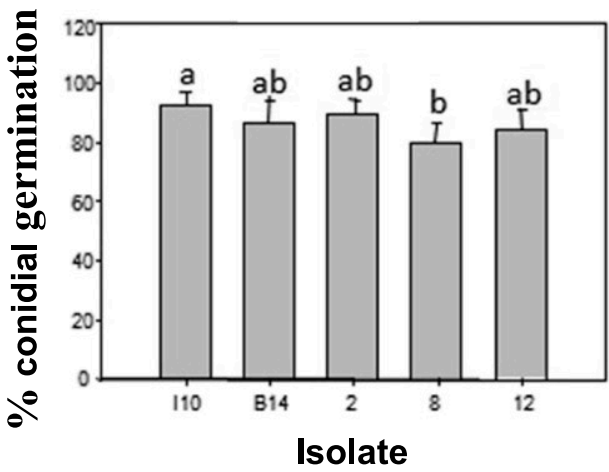

D

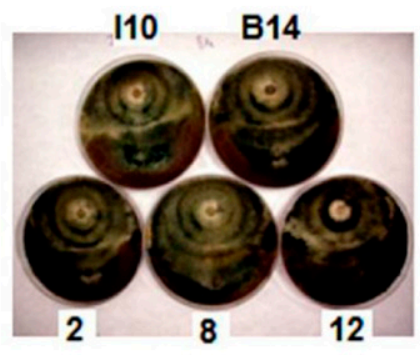

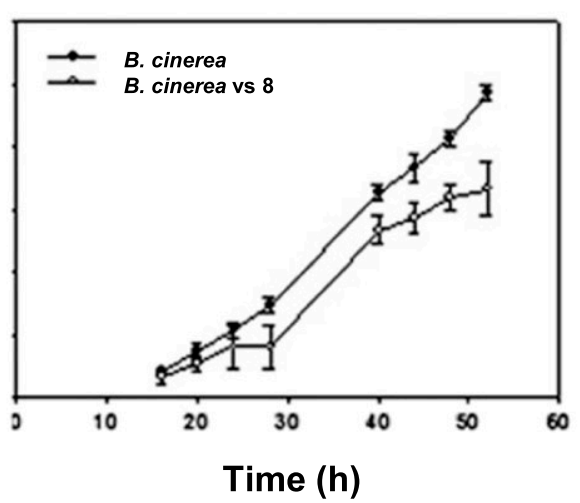

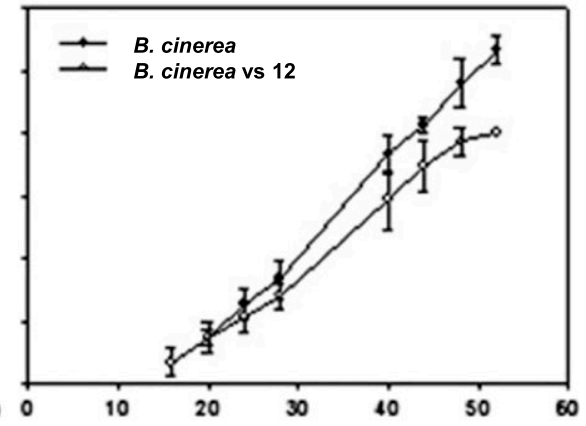

Time ( $\mathrm{h}$ )

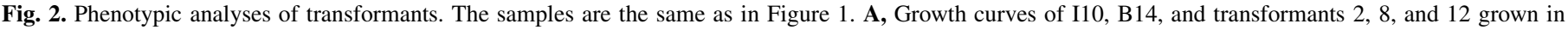

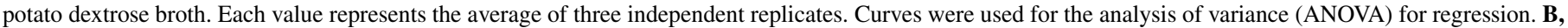

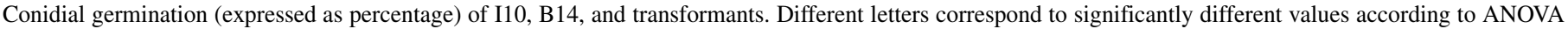

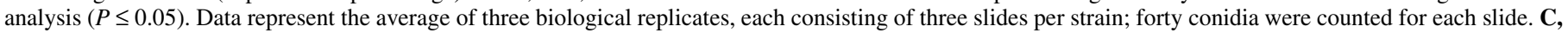

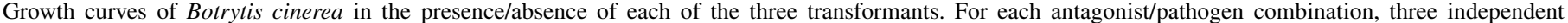
replicates were made. Curves were used for the ANOVA for regression. D, Overgrowth and sporulation of I10, B14, and transformants on B. cinerea. 
earlier in strain I10 (Baroncelli et al. 2016). Instead, none of the transcripts were present in the $\Delta t v p g 2$ knockout mutant (Fig. 3C), suggesting that tvpg 2 plays a regulatory role in tvpg 1 expression.

The fungal growth on different media was also evaluated. The biomasses obtained from strains I10, B14, and $\Delta t v p g 2$ in each liquid inducing medium were measured, and no significant differences were detected among the three fungi in any condition (data not shown).

TvPG2 plays an essential role in the expression of T. virens tvpg1 and tomato Lepgpil during the interaction with tomato roots. To investigate the consequences of tvpg2 deletion on the interaction between $T$. virens and tomato roots, we tested the expression of tvpg 1, encoding the inducible EPG (Baroncelli et al. 2016), and of tomato Lepgpi1, which encodes an PGIP. To this end, tomato roots were in vivo-inoculated with I10wt, B14 or $\Delta t v p g 2$ for 24,48 , and $72 \mathrm{~h}$. T. virens strains (I10, B14, and $\Delta t v p g 2$ ) in the soil without plants, and noninoculated roots were grown as controls. Consistent with earlier findings (Catalano et al. 2011), the I10 isolate constitutively expressed tvpg2, whereas tvpg 1 was induced with a peak at $48 \mathrm{~h}$ (Fig. 4A), and the Lepgip1 expression paralleled that of tvpg 1 with highest levels at $48 \mathrm{~h}$ (Fig. 4B). As expected, the B 14 expression pattern overlapped the pattern shown by $\mathrm{I} 10$ (Fig. 4A and B). On the other hand, during the interaction with tomato roots the expression of all $\Delta$ tvpg 2 genes exhibited a different pattern compared with $\mathrm{I} 10$ and B14 genes. The expected lack of tvpg 2 transcripts was evident; however, tvpg 1 and Lepgipl were not expressed either (Fig. 4A and B). These results suggest that TvPG2 plays a regulatory role in the induction of tvpg1, and as a consequence is also crucial for the expression of Lepgipl.

Induction of systemic resistance in tomato. The results described so far prompted us to investigate whether TvPG2 is necessary for triggering an ISR in plants. Tomato plants were thus in vivo-preinoculated with the T. virens strains I10, B 14, or $\Delta t v p g 2$ and after 1 week plants were inoculated by $B$. cinerea. Specific $B$. cinerea necrotic symptoms occurring on plant leaves were evaluated 15 days after infection (dpi). In order to quantify the plant response, the number of lesions per leaf was recorded for all inoculated leaves present on plants. The resulting data (Table 2) revealed a significant difference between I10/B14 and $\Delta t v p g 2$, and all three were in turn significantly dissimilar to the $B$. cinerea control (in all cases $P<$ 0.001 ). Evidently, the induction of the resistance mechanism prompted by $\mathrm{I} 10$ and B14 strains in the plant is strongly reduced (about 100-fold) using the mutant $\Delta t v p g 2$. Pretreating roots with the strain $\Delta t v p g 2$ still resulted in some protection of the tomato against $B$. cinerea (about five times less than the B. cinerea control) revealing, however, that TvPG2 is not the only signal of $T$. virens that acts as an elicitor of ISR.

Root and rhizosphere colonization. The ability of I10, B14 and $\Delta t v p g 2$ to grow in the tomato roots or to colonize the rhizosphere was evaluated on 2-week-old plants, 7 days after inoculation of the roots. Observations were made 2 weeks after plating roots and rhizosphere suspensions on TSM. The plant growing substrate itself was also plated on TSM and no T. virens colonies were observed. The few Trichoderma colonies present had a clearly different morphology compared with $T$. virens $\mathrm{I} 10$ when grown on the same medium (data not shown). Moreover, no Trichoderma colonies were observed also in plates seeded by roots or rhizosphere suspensions derived from control plants, suggesting that the isolates naturally present in the plant growing substrate were unable to grow in the roots and unable to colonize the rhizosphere $\left(\mathrm{H}_{2} \mathrm{O}\right.$ ctrl, Table 3$)$.

From this semiquantitative analysis, no differences in the percentage of plant roots colonized by each strain was detected. The morphology of all the developed colonies was similar to that of
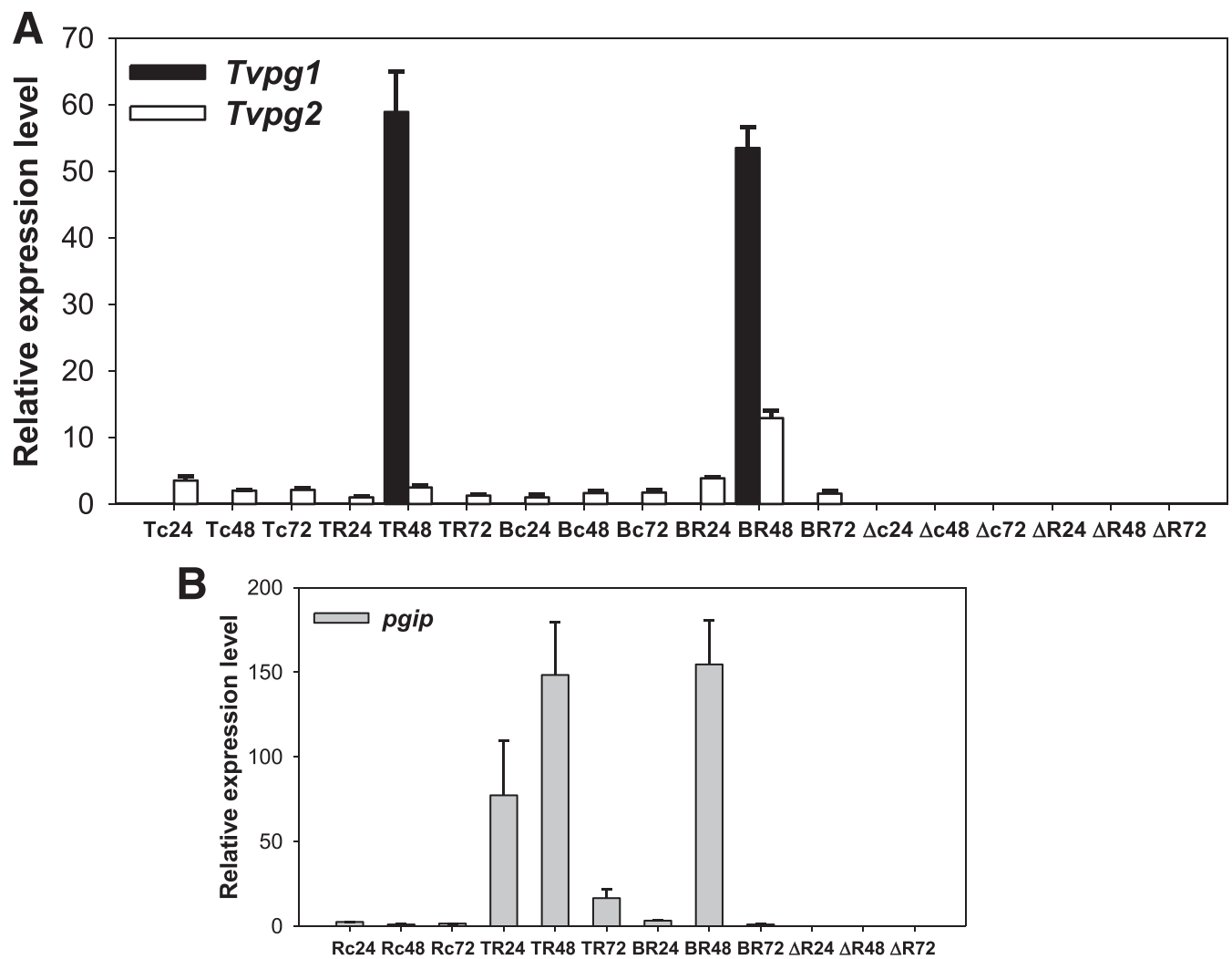

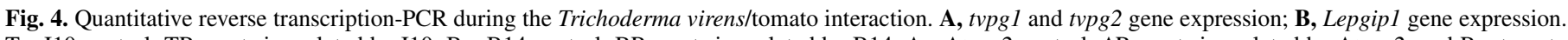

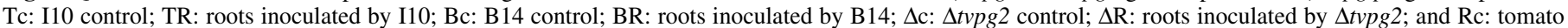

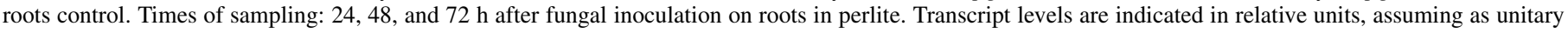

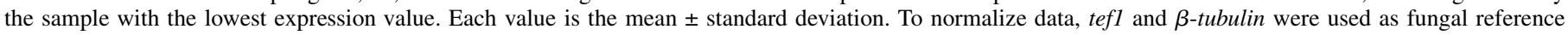

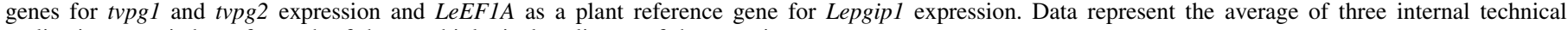
replications, carried out for each of the two biological replicates of the experiment. 
a pure colony of $T$. virens I10 grown for the same time on TSM. The ability of $T$. virens $\mathrm{I} 10$ and its mutants to grow in plant roots is, therefore, very limited $(11 \%)$ and is not affected by the mutations investigated in this paper. Instead T. virens was recorded on all plates $(100 \%)$ derived from the rhizosphere of plants inoculated with I10, B14, and $\Delta t v p g 2$ (Table 3). These results confirm that I10 is a good rhizosphere competent isolate and not an endophyte. This suggests that mutations in B14 and in $\Delta$ tvpg 2 do not modify the relationships established between $\mathrm{I} 10$ and roots/rhizosphere of tomato plants.

\section{DISCUSSION}

Some Trichoderma spp. colonize the rhizosphere and establish interactions with plant roots, although the physical association is usually limited to the outermost external layers (Harman et al. 2004). This association triggers a molecular cross-talk between the two organisms which leads to reciprocal beneficial events, such as nutrients supply for Trichoderma and growth promotion, or immunity against pathogens in plant. Pectinases, and especially EPGs, are required by plant pathogenic fungi to gain access to the host. In turn, plants recognize the presence of pectinases and activate appropriate plant defense responses (Cervone et al. 1989).

In contrast to other plant cell wall degrading enzymes (Enkerli et al. 1999), the activity of EPGs is essential for this stimulation, since plant cell wall-derived oligogalacturonides within a limited range of polymerization, function as elicitors of plant responses (Cervone et al. 1989). The release of these oligomers initiates a PGIP mediated signaling mechanism and elicits defense reactions (Federici et al. 2006). PGIPs thereby reduce the pectinase activity, which would otherwise continue to degrade the oligomers and produce molecules that are too short to function as elicitors (D'Ovidio et al. 2004).

Previous in vivo investigations on tomato roots inoculated by T. virens showed the expression of two EPGs (an inducible gene, tvpg 1 , and a constitutive gene tvpg2), and the induction of a plant PGIP encoding gene, Lepgip1, which paralleled the expression of tvpg1 (Baroncelli et al. 2016). These data suggested the activity of an EPG-PGIP recognition system. In addition, the availability of both constitutive and inducible EPGs in $T$. virens suggests the potential for different functionalities during its interaction with plants, as reported elsewhere (Kemp et al. 2004). In fungi, the presence of constitutively expressed EPGs has been linked to aggressiveness and pathogenic potential against plants (Shieh et al. 1997; Wubben et al. 1999). It may shorten the time of contact with the plant and allow the pectin methyl esterase to degrade the esterified pectin and thus decrease the $\mathrm{pH}$ thereby triggering the expression of the inducible genes (Kemp et al. 2004). In this regard it is notable that, with the exception of T. atroviride, TvPG2 has no ortholog in other Trichoderma spp. Proteins with the highest similarity were found in Fusarium spp. (C. P. Kubicek, unpublished data), suggesting its possible origin as a result of horizontal gene transfer. This would explain the "pathogen-like" behavior of the constitutive TvPG2 toward plants. Furthermore, the alkaline isoelectric point of TvPG2, theoretical 9.6, fits models where a

TABLE 2. Botrytis cinerea disease severity on tomato leaves ${ }^{\mathrm{x}}$

\begin{tabular}{lcc}
\hline Treatment & Lesions $^{\mathrm{y}}$ & Total leaves $^{\mathrm{z}}$ \\
\hline B. cinerea ctrl & $5.12 \pm 3.14 \mathrm{a}^{* * *}$ & 107 \\
B. cinerea $+\mathrm{I} 10$ & $0.01 \pm 0.08 \mathrm{c}$ & 145 \\
B. cinerea $+\mathrm{B} 14$ & $0.01 \pm 0.11 \mathrm{c}$ & 141 \\
B. cinerea $+\Delta$ tvpg 2 & $0.96 \pm 1.52 \mathrm{~b}$ & 121 \\
\hline
\end{tabular}

x Plants infected by $B$. cinerea (15 days after inoculation) without any pretreatment (ctrl), or preinoculated by $\mathrm{I10}, \mathrm{B} 14$, or $\Delta t v p g 2$.

y Averaged numbers of lesions ( \pm standard deviation) per leaf, resulting from three replicates each consisting of two pots, three plants per pot. Different letters correspond to significantly different values $(P<0.001)$, according to analysis of variance.

${ }^{\mathrm{z}}$ Number of leaves observed for each treatment, corresponding to the sum of inoculated leaves in all replicates. constitutive enzymatic activity opens the plant cell wall and thus provides access for other degrading enzymes working at a lower local $\mathrm{pH}$, such as TvPG1 which has a theoretical isoelectric point of 6.3 (Baroncelli et al. 2016; Kemp et al. 2004).

Our study showed, both in vitro and in vivo, that the inducible tvpg1 is also not expressed in the absence of TvPG2, thus indicating a regulative action by the constitutive tvpg 2 on tvpg 1 . In addition, the deletion of tvpg 2 leads to a simultaneous loss of tvpg1 and Lepgip1 transcription during the interaction between $T$. virens and tomato. This outcome confirms the coordinated action of the two fungal genes and the plant gene under analysis, implying that TvPG2 is needed in order to induce the expression of both tvpgl and Lepgipl.

These findings may be explained by hypothesizing that the constitutive TvPG2 works at the first contact with plant roots and consequently triggers the expression of tvpgl in T. virens and of Lepgipl in tomato. For the moment it is unclear whether tvpg2 induces Lepgipl directly (by releasing oligogalacturonides) or indirectly (by activating the expression of tvpgl which in turn releases oligogalacturonides).

Structural properties of EPGs have been widely studied and the target amino acids crucial for the PGIP recognition, the formation of an EPG-PGIP complex and therefore EPG inhibition by PGIP have been identified (Raiola et al. 2008; Stotz et al. 2000). Note that both T. virens TvPG1 and TvPG2 (Baroncelli et al. 2016) contain, at fixed positions in relation to the active site, the three amino acid residues that are strictly required for interacting with PGIPs in Fusarium moniliforme pectinases, i.e., H188, R267, and K269, (Federici et al. 2001). This in silico result is consistent with the potential ability of TvPG1 and TvPG2 to recognize a plant PGIP.

In order to verify whether the PGIP activation by $T$. virens in tomato is able to also elicit a plant immune response, a three-way plant-pathogen-antagonist interaction system has been developed. Trichoderma-plant-pathogen interactions have already been used for monitoring the induction of plant resistance (Howell et al. 2000), for the analysis of the T. atroviride proteome (Marra et al. 2006), and to study the function of selected Trichoderma genes during biological control (Mukherjee et al. 2012; Vinale et al. 2008). With regards to the latter, a gene encoding an inducible EPG, Thpg 1, was silenced in T. harzianum and shown to be involved in tomato root colonization. However, and unlike the present study, in vivo biocontrol assays on plants infected with $B$. cinerea did not show significant differences between the wild-type and the Thpgl silenced strain (Morán-Diez et al. 2009). In our approach, tomato roots had been in vivo inoculated by $T$. virens and leaves were then sprayed by $B$. cinerea. The effective systemic response of the tomato plants to $B$. cinerea infection, which was promoted by root pretreatment with the $T$. virens wild-type strain, was strongly impaired when the $T$. virens mutant with a deleted tvpg 2 was used. These data again underline the importance of constitutively expressed EPGs and confirm the crucial role of the tvpg 2 gene in triggering the PGIP signaling pathway, which in turn activates an ISR response in plants.

TABLE 3. Colonization of tomato roots or rhizosphere by Trichoderma virens strains $^{\mathrm{Z}}$

\begin{tabular}{lcc}
\hline Treatment & Roots $(\%)$ & Rhizosphere $(\%)$ \\
\hline $\mathrm{H}_{2} \mathrm{O}$ ctrl & 0 & 0 \\
$\mathrm{I} 10$ & 11 & 100 \\
$\mathrm{~B} 14$ & 11 & 100 \\
Atvpg2 & 11 & 100 \\
\hline
\end{tabular}

z Plant roots, 1-week-old, were inoculated by I10, B14, or $\Delta t v p g 2$ and by water (ctrl). Roots: samples rinsed, removed from the plant, sterilized, cut in pieces, and laid on Trichoderma semiselective medium (TSM) plates. Rhizosphere: roots washing off spread on TSM plates. All samples were observed up to 2 weeks after plating for checking the presence of T. virens. Data represent the percentage of roots or rhizosphere samples giving rise to $T$. virens colonies. Three independent replicates, each consisting of two pots per treatment, each one containing three plants, were tested. 
Data regarding the root and the rhizosphere colonization showed no difference in behavior between the mutant and the two control strains. This observation, although within the limits of a semiquantitative technique, suggests that there is no interference of a possible impaired colonization with the reduced induction of the plant response by $\Delta t v p g 2$. Furthermore, the poor ability to penetrate into plant roots and the high rhizosphere competence shown by I10 isolate is consistent with other reports on rhizosphere competent fungi inducing systemic resistance without needing to be endophytic (Druzhinina et al. 2011; Harman et al. 2004).

In conclusion we believe that our investigation contributes to understanding the interaction of a biocontrol agent with a plant and how it modulates the plant reaction to a pathogen attack. The data set reported shows a coordinated regulation between the two T. virens EPGs and a tomato PGIP, which results in the activation of ISR against $B$. cinerea. All these events are dependent on the presence of the constitutive tvpg2, which appears to regulate the cross-talk between $T$. virens and tomato, mimicking the behavior of a fungal plant pathogen. Given the importance of eliciting systemic plant defense responses as an antagonistic mechanism versus pathogens, the beneficial effects of this T. virens isolate and the role of its TvPG2 offer strategies for further improvements toward successful biological control.

\section{ACKNOWLEDGMENTS}

We thank S. Gruber (at the Institute of Chemical Engineering, TU Vienna, Austria) for her assistance in the T. virens transformations.

\section{LITERATURE CITED}

Annis, S. E., and Goodwin, P. H. 1997. Recent advances in the molecular genetics of plant cell wall-degrading enzymes produced by plant pathogenic fungi. Eur. J. Plant Pathol. 103:1-14.

Baroncelli, R., Matarese, F., Moncini, L., Vannacci, G., and Vergara, M. 2016. Two endopolygalacturonase genes in Trichoderma virens: In silico characterisation and expression during interaction with plant. J. Phytopathol. 164:18-28.

Benitez, T., Rincòn, A. M., Limòn, M. C., and Codòn, A. C. 2004. Biocontrol mechanisms of Trichoderma strains. Int. Microbiol. 7:249-260.

Catalano, V., Vergara, M., Hauzenberger, J. R., Seiboth, B., Sarrocco, S., Vannacci, G., Kubicek, C. P., and Seidl-Seiboth, V. 2011. Use of a nonhomologous end-joining-deficient strain (delta-ku70) of the biocontrol fungus Trichoderma virens to investigate the function of the laccase gene lcc1 in sclerotia degradation. Curr. Genet. 57:13-23.

Cervone, F., Hahn, M. G., De Lorenzo, G., Darvill, A., and Albersheim, P. 1989. Host-pathogen interactions XXXIII. A plant protein converts a fungal pathogenesis factor into an elicitor of plant defense responses. Plant Physiol. 90:542-548.

Contreras-Cornejo, H. A., Macias-Rodríguez, L., Cortés-Penagos, C., and López-Bucio, J. 2009. Trichoderma virens, a plant beneficial fungus, enhances biomass production and promotes lateral root growth through an auxin dependent mechanism in Arabidopsis. Plant Physiol. 149:1579-1592.

D'Ovidio, R., Mattei, B., Roberti, S., and Bellincampi, D. 2004. Polygalacturonases, polygalacturonase-inhibiting proteins and pectic oligomers in plant-pathogen interactions. Biochim. Biophys. Acta 1696:237-244.

De Meyer, G., Bigirimana, J., Elad, Y., and Hofte, M. 1998. Induced systemic resistance in Trichoderma harzianum T39 biocontrol of Botrytis cinerea. Eur. J. Plant Pathol. 104:279-286.

Di Pietro, A., and Roncero, M. I. G. 1998. Cloning, expression, and role in pathogenicity of $\mathrm{pgl}$ encoding the major extracellular endopolygalacturonase of the vascular wilt pathogen Fusarium oxysporum. Mol. PlantMicrobe Interact. 11:91-98.

Druzhinina, I. S., Seidl-Seiboth, V., and Herrera-Estrella, A. 2011. Trichoderma: The genomics of opportunistic success. Nat. Rev. Microbiol. 9:749-759.

Elsharkawy, M. M., Shimizu, M., Takahashi, H., Ozaki, K., and Hyakumachi, M. 2013. Induction of systemic resistance against Cucumber mosaic virus in Arabidopsis thaliana by Trichoderma asperellum SKT-1. Plant Pathol. J. 29:193-200

Enkerli, J., Felix, G., and Boller, T. 1999. The enzymatic activity of fungal xylanase is not necessary for its elicitor activity. Plant Physiol. 121: 391-398.

Federici, L., Caprari, C., Mattei, F., Savino, C., Di Matteo, A., De Lorenzo, G., Cervone, F., and Tsernoglou, D. 2001. Structural requirements of endo- polygalacturonase for the interaction with PGIP (polygalacturonaseinhibiting protein). Proc. Natl. Acad. Sci. USA 98:13425-13430.

Federici, L., Di Matteo, A., Fernandez-Recio, J., Tsernoglou, D., and Cervone, F. 2006. Polygalacturonase inhibiting proteins: Players in plant innate immunity? Trends Plant Sci. 11:65-70.

Fiorini, L., Guglielminetti, L., Mariotti, L., Curadi, M., Picciarelli, P., Scartazza, A., Sarrocco, S., and Vannacci, G. 2016. Trichoderma harzianum T6776 modulates a complex metabolic network to stimulate tomato cv. Micro-Tom growth. Plant Soil 400:351-366.

Harman, G. E., Howell, C. R., Viterbo, A., Chet, I., and Lorito, M. 2004. Trichoderma species-opportunistic, avirulent plant symbionts. Nat. Rev. Microbiol. 2:43-56.

Hartl, L., Kubicek, C. P., and Seiboth, B. 2007. Induction of the gal pathway and cellulase genes involves no transcriptional inducer function of the galactokinase in Hypocrea jecorina. J. Biol. Chem. 282:18654-18659.

Hartl, L., and Seiboth, B. 2005. Sequential gene deletions in Hypocrea jecorina using a single blaster cassette. Curr. Genet. 48:204-211.

Hermosa, R., Viterbo, A., Chet, I., and Monte, E. 2012. Plant-beneficial effects of Trichoderma and of its genes. Microbiology 158:17-25.

Herron, S. R., Benen, J. A. E., Kester, H. C. M., and Visser, J. 2000. Structure and function of pectic enzymes: virulence factors of pathogens. Proc. Natl. Acad. Sci. USA 97:8762-8769.

Howell, C. R., Hanson, L. E., Stipanovic, R. D., and Puckhaber, L. S. 2000. Induction of terpenoid synthesis in cotton roots and control of Rhizoctonia solani by seed treatment with Trichoderma virens. Phytopathology 90:248-252.

Kemp, G., Stanton, L., Bergmann, C. W., Clay, R. P., Albersheim, P., and Darvill, A. 2004. Polygalacturonase-inhibiting proteins can function as activators of polygalacturonase. Mol. Plant-Microbe Interact. 17:888-894.

Kubicek, C. P., Herrera-Estrella, A., and Seidl-Seiboth, V. 2011. Comparative genome sequence analysis underscores mycoparasitism as the ancestral life style of Trichoderma. Genome Biol. 12:R40.

Kubicek, C. P., Mach, R. L., Peterbauer, C. K., and Lorito, M. 2001. Trichoderma: From genes to biocontrol. J. Plant Pathol. 83:11-23.

Kubicek, C. P., Starr, T. L., and Glass, N. L. 2014. Plant cell wall-degrading enzymes and their secretion in plant-pathogenic fungi. Annu. Rev. Phytopathol. 52:427-451.

Lotan, T., and Fluhr, R. 1990. Xylanase, a novel elicitor of pathogenesisrelated proteins in tobacco, uses a non-ethylene pathway for induction. Plant Physiol. 93:811-817.

Marra, R., Ambrosino P., Carbone, V., Vinale, F., Woo, S. L., and Ruocco, M. 2006. Study of the three-way interaction between Trichoderma atroviride, plant and fungal pathogens by using a proteomic approach. Curr. Genet. 50: 307-321.

Morán-Diez, E., Hermosa, R., Ambrosino, P., Cardoza, R. E., Gutiérrez, S., Lorito, M., and Monte, E. 2009. The thpgl endopolygalacturonase is required for the Trichoderma harzianum-plant beneficial interaction. Mol. Plant-Microbe Interact. 22:1021-1031.

Mukherjee, M., Mukherjee, P. K., Horwitz, B. A., Zachow, C., Berg, G., and Zeilinger, S. 2012. Trichoderma-plant-pathogen interactions: Advances in genetics of biological control. Indian J. Microbiol. 52:522-529.

Raiola, A., Sella, L., Castiglioni, C., Balmas, V., and Favaron, F. 2008. A single amino acid substitution in highly similar endo-PGs from Fusarium verticillioides and related Fusarium species affects PGIP inhibition. Fungal Genet. Biol. 45:776-789.

Salas-Marina, M. A., Silva-Flores, M. A., Uresti-Rivera, E. E., Castro-Longoria, E., Herrera-Estrella, A., and Casas-Flores, S. 2011. Colonization of Arabidopsis roots by Trichoderma atroviride promotes growth and enhances systemic disease resistance through jasmonic acid/ethylene and salicylic acid pathways. Eur. J. Plant Pathol. 131:15-26.

Sambrook, J., and Russell, D. W. 2001. Molecular Cloning: A Laboratory Manual. 2nd ed. Cold Spring Harbor Laboratory, Cold Spring Harbor, NY.

Sarrocco, S., Guidi, L., Fambrini, S., Degl'Innocenti, E., and Vannacci, G. 2009. Competition for cellulose exploitation between Rhizoctonia solani and two Trichoderma isolates in the decomposition of wheat straw. J. Plant Pathol. 91:331-338.

Sarrocco, S., Mikkelsen, L., Vergara, M., Jensen, D. F., Lübeck, M., and Vannacci, G. 2006. Histopathological studies of sclerotia of phytopathogenic fungi parasitized by a GFP transformed Trichoderma virens antagonistic strain. Mycol. Res. 110:179-187.

Shieh, M.-T., Brown, R. L., Whitehead, M. P., Cary, J. W., Cotty, P. J., Cleveland, T. E., and Dean, R. A. 1997. Molecular genetic evidence for the involvement of a specific polygalacturonase, P2c, in the invasion and spread of Aspergillus flavus in cotton bolls. Appl. Environ. Microbiol. 63: 3548-3552.

Shoresh, M., Harman, G. E., and Mastoury, F. 2010. Induced systemic resistance and plant responses to fungal biocontrol agents. Annu. Rev. Phytopathol. 48:21-43.

Stotz, H. U., Bishop, J. G., Bergmann, C. W., Koch, M., Albersheim, P., Darvill, A. G., and Labavitch, J. M. 2000. Identification of target amino 
acids that affect interactions of fungal polygalacturonases and their plant inhibitors. Physiol. Mol. Plant Pathol. 56:117-130.

Vannacci, G., and Pecchia, S. 2000. Discovery of Trichoderma I252 and Gliocladium I10, components of the biofungicide GT10/252. European Cost 830 Workshop 'Selection Strategies for Plant Beneficial Microorganisms', Nancy, 3-5 April 2000.

Velázquez-Robledo, R., Contreras-Cornejo, H. A., Macías-Rodríguez, L., Hernández-Morales, A., Aguirre, J., Casas-Flores, S., López-Bucio, J., and Herrera-Estrella, A. 2011. Role of the 4-phosphopantetheinyl transferase of Trichoderma virens in secondary metabolism and induction of plant defense responses. Mol. Plant-Microbe Interact. 24:1459-1471.
Vinale, F., Sivasithamparam, K., Ghisalberti, E. L., Marra, R., Woo, S. L., and Lorito, M. 2008. Trichoderma-plant-pathogen interactions. Soil Biol. Biochem. 40:1-10. Wubben, J. P., Mulder, W., Ten Have, A., Van Kan, J. A. L., and Visser, J. 1999. Cloning and partial characterization of endopolygalacturonase genes from Botrytis cinerea. Appl. Environ. Microbiol. 65:1596-1602.

Yedidia, I., Benhamou, N., and Chet, I. 1999. Induction of defense responses in cucumber plants (Cucumis Sativus L.) by the biocontrol agent Trichoderma harzianum. Appl. Environ. Microbiol. 65:1061-1070.

Zhao, Z., Liu, H., Wang, C., and Xu, J. R. 2013. Comparative analysis of fungal genomes reveals different plant cell wall degrading capacity in fungi. BMC Genomics 14:274. 